Published in final edited form as:

Curr Opin Cardiol. 2015 May ; 30(3): 228-234. doi:10.1097/HCO.0000000000000159.

\title{
Adeno-associated virus-mediated gene therapy in cardiovascular disease
}

\author{
Nadjib Hammoudi, Kiyotake Ishikawa, and Roger J. Hajjar \\ Cardiovascular Research Center, Icahn School of Medicine at Mount Sinai, New York, New York, \\ USA
}

\section{Abstract}

Purpose of review-The use of adeno-associated virus (AAV) as an efficient, cardiotropic, and safe vector, coupled with the identification of key molecular targets have placed gene-based therapies within reach of cardiovascular diseases. The purpose of this review is to provide a focused update on the current advances related to AAV-mediated gene therapy in cardiovascular diseases and particularly in heart failure (HF) wherein gene therapy has recently made important progress.

Recent findings-Multiple successful preclinical studies suggest a potential utility of AAV gene therapy for arrhythmias and biological heart pacing, as well as RNA overexpression. Moreover, AAV-mediated overexpression of several molecular targets involved in HF has demonstrated promising results in clinically relevant large animal models. In human, a safe and successful completion of a phase 2 clinical trial targeting the sarcoplasmic reticulum calcium ATPase pump with AAV has been reported. Serial studies are ongoing to further prove the efficacy of AAV-mediated sarcoplasmic reticulum calcium ATPase pump gene transfer in human HF.

Summary-Significant progress in clinical translation of AAV-mediated cardiac gene therapy has been achieved in recent years. This will prompt further clinical trials, and positive results could open a new era for cardiac gene therapy.

\section{Keywords}

adeno-associated virus; arrhythmias; gene therapy; heart failure; sarcoplasmic reticulum calcium ATPase pump

\section{INTRODUCTION}

Cardiovascular disease is a major cause of morbidity and mortality in the United States [1]. Despite optimal guideline-directed therapy employing a wide range of pharmacological, device, and surgical options, many patients deteriorate over time and develop refractory

Copyright () 2015 Wolters Kluwer Health, Inc. All rights reserved.

Correspondence to Roger J. Hajjar, Cardiovascular Research Center, Icahn School of Medicine at Mount Sinai, One Gustave L Levy Place, Box, 1030, New York, NY 10029, USA. Tel: +1 212241 8374; fax: +1 212241 4080; roger.hajjar@mssm.edu.

Conflicts of interest

R.J.H. is a cofounder of Celladon, which is developing AAV1.SERCA2a for the treatment of heart failure. 
symptoms [2]. Considering the medical, economic, and quality-of-life consequences of these trends, novel treatment strategies for cardiovascular diseases, especially those aimed at reducing hospitalizations, are needed.

Gene therapy was initially applied clinically for inherited monogenic disorders. It is now apparent that gene therapy has a broader potential for acquired diseases such as congestive heart failure (HF). Improvement in our understanding of the molecular mechanisms in cardiovascular diseases has led to the identification of novel targets that are difficult to manipulate pharmacologically, but may be more amenable to gene therapy.

The application of adeno-associated viral (AAV) vectors has accelerated the translation of gene therapy to the cardiac field because AAVs confer prolonged gene expression after a single delivery compared with adenovirus or plasmid-based gene delivery approaches [3]. Furthermore, the lack of human disease caused by AAV makes them an attractive tool for clinical cardiac gene therapy [4]. Indeed, a first-in-human clinical trial targeting HF with virus-mediated overexpression of sarcoplasmic reticulum calcium ATPase (SERCA2a) pump employed AAV serotype 1 [5] has shown to be safe and effective in phase 1 and phase 2 trials $[6,7 \mathbf{r}]$.

The purpose of this review is to provide a focused update on the current advances related to AAV-mediated gene therapy in cardiovascular diseases and particularly in the HF field in which gene therapy has recently made important progress.

\section{ADENO-ASSOCIATED VIRUS FOR GENE THERAPY}

The appropriate combination of specific vectors and therapeutic genes are key for successful gene therapy. AAV had originally been identified as a contamination in Simian adenovirus preparations [8]. AAV is part of the parvovirus family and is a small, nonpathogenic human virus with a nonenveloped capsid and single-stranded DNA [4]. Of the 13 serotypes known so far, AAV 1, 6, 8, and 9 have shown cardiac tropism [9]. There are several reasons why AAV vectors are increasingly used as gene therapy vectors in the setting of cardiovascular diseases. First, recombinant AAVs used for gene therapy are nonpathogenic in humans and do not integrate in the host genome [4]. Second, AAVs are minimally immunogenic especially when compared with adenovirus [10]. Third, the small size of AAV is an advantage as it allows for their delivery to the myocardium by infusion through the coronary arteries; and finally, AAV vectors are able to induce long-term transgene expression in nondividing cells after a single delivery [11].

One of the main drawbacks of using AAV in gene therapy applications is the limited packaging capacity of $\sim 4.7 \mathrm{~kb}$ [4]. To overcome this limitation, trans-splicing based or recombination-based overlapping AAV vector approaches have increased the gene delivery capacity. Two independent AAV vectors each carrying parts of a transgene with appropriate splice signals or with overlapping sequence elements are independently packaged in AAV vectors [12]. More recently, a triple AAV vector coinfection was tested with success in mice to reconstitute the functional, full-length dystrophin coding sequence [13]. Whether these approaches are effective in cardiac gene transfer remains to be evaluated in future studies. 
The fact that many AAV serotypes appear to be endemic, results in extensive antiviral immunity in human populations [14]. From a clinical point of view, this issue is very significant because the presence of neutralizing antibodies against the AAV serotype limits the potential clinical candidates for the gene therapy [15-]. Over the past years, significant effort has been put into the development of AAV variants that circumvent the effect of neutralizing antibodies $[14,16]$. Recently, a chimeric AAV vector developed by capsid reengineering was shown to effectively transduce the heart for the first time in a clinically relevant large animal model [17-]. Additionally, plasmapheresis has shown encouraging results in preclinical studies to reduce the effect of antibodies [14,18].

\section{ADENO-ASSOCIATED VIRUS CARDIAC DELIVERY METHODS}

The vector delivery method is another important factor for successful cardiac gene transfer. Although intravenous injection is effective to transduce the heart in mice, more advanced animals require cardiac-specific delivery even with the cardiotropic AAV vectors. In the first-in-human clinical trial of gene therapy in the setting of HF, antegrade intracoronary injection was the choice for AAV delivery because of its relatively safe, simple, and minimally invasive nature [5]. Several different approaches are proposed to increase the transduction efficacy and cardiac specificity using large animals. Retrograde delivery into the coronary sinus was tested in pigs using AAV-6 with successful transduction to the heart [19]. There is also a surgical recirculation delivery method, which was recently applied to deliver AAV-9 SERCA2a in a sheep model that resulted in positive improvement in the left ventricular function [20-]. This approach may reduce the extracardiac gene expression, and at the same time circumvent the antibody inhibition by AAV. Although this approach is more invasive than catheter-based delivery, it may be first applied to patients who are scheduled for bypass surgery or valve replacement surgery to establish its clinical utility. The same group also reported a novel method to deliver AAV by liquid jet injection [21]. Although safety and efficacy of these delivery methods need to be further established in future studies, such attempts for novel delivery approaches will certainly advance the cardiac gene therapy field. The optimal clinically translatable technique for global cardiomyocyte delivery has yet to be developed and the ideal delivery method is likely to vary depending on the target gene.

\section{MOLECULAR TARGETS}

AAV-mediated overexpression of several molecular targets has demonstrated promising results in cardiovascular disease.

\section{Sarcoplasmic reticulum calcium ATPase pump}

Abnormal calcium cycling is a common characteristic in patients with advanced HF regardless of cause. Both the amplitude and decay rate of the intracellular calcium transient are blunted in cells and tissues from failing hearts [22]. SERCA2a plays a pivotal role in calcium handling and numerous studies have shown that SERCA2a expression is decreased in HF, whereas restoring the SERCA2a expression level improves cardiac function [23]. Supported by extensive preclinical studies focusing on efficacy and safety of SERCA2a 
gene therapy using an AAV vector, this approach is currently being investigated in humans. The results of clinical trials are discussed later in this review [6,7-].

Abnormal calcium cycling plays a key role in the pathophysiology of vascular remodeling and SERCA2a is downregulated. Recently, another beneficial effect of AAV-1 mediated SERCA2a gene therapy on the vasculature was focused and investigated. In a pig model of mitral regurgitation, AAV-1 mediated SERCA2a gene transfer increased coronary flow through enhanced endothelial nitric oxide synthase expression in endothelial cells [24]. Furthermore, aerosol delivery of AAV-1 SERCA2a in a mouse model of monocrotalineinduced pulmonary arterial hypertension decreased pulmonary arterial remodeling and suppressed right ventricular remodeling [25-]. Further study in more advanced species is awaited to enable translation of this promising approach into clinics.

\section{Small ubiquitin-like modifier 1}

AAV-based experiments have proven the important role of proteins associated with SERCA2a. A recent study has brought to light the interaction between SERCA2a and the small ubiquitin-related modifier 1 (SUMO-1) through a posttranscriptional modification process called SUMOylation [26]. The amount of myocardial SUMO-1 is decreased in failing hearts, and its knockdown results in severe HF in mice [27]. SUMO-1 was shown to preserve SERCA2a function and stability, and the overexpression of SUMO1 in a rodent model of HF had favorable effects on myocardial function [26]. AAV-9 gene transfer of SUMO-1 prevented the heart from undergoing hypertrophy after transverse aortic constriction and prevented the development of left ventricular dysfunction in mice. Furthermore, SUMO-1 expression protects SERCA2a from oxidative stress [28]. Toward clinical translation, a recent study evaluated the effects of SUMO-1 gene transfer using AAV-1 in a swine model of ischemic HF. Compared with control animals, SUMO-1 gene transfer and its combination with SERCA2a improved cardiac function and halted left ventricular remodeling [29-]. These results support the critical role of SUMO-1 in SERCA2a regulation and underline the therapeutic potential of SUMO-1 for HF patients.

\section{S100A1}

The calcium sensor protein S100A1 has emerged as an attractive target for HF gene therapy because of its characteristic molecular profile [30]. The S100A1 protein regulates a network in cardiomyocytes that controls sarcoplasmic reticulum calcium cycling and mitochondrial function through interaction with the ryanodine receptor, SERCA2a, and mitochondrial F1ATPase activity, causing antihypertrophic, positive inotrope, and antiarrhythmic effects and reducing energy depletion in HF [31]. Recently, large animal studies in a swine model of HF showed the feasibility, therapeutic efficacy, and safety of AAV-6-mediated and AAV-9mediated S100A1 gene therapy [32,33-]. Clinical trials with AAV9.S100A1 are being planned in the coming few years.

\section{Protein phosphatase 1 inhibitor-1}

The decrease in activity of SERCA2a observed in HF is at least partially attributable to enhanced phospholamban (PLN) inhibition. It has been found that protein phosphatase 1 activity serves to regulate the phosphorylation pattern of PLN [34]. Expression of protein 
phosphatase 1 is elevated in HF, limiting the deactivation of PLN, and this is associated with decreased expression of inhibitor-1 (Fig. 1) [34,35]. Overexpression of the constitutively active, truncated form of inhibitor-1(I-1c) in the heart results in significant enhancement of PLN phosphorylation that results in increased cardiac function and abrogation of the negative effects in small animal aortic constriction models [36]. Recent studies report improved cardiac function after I-1c gene transfer in clinically relevant models of postmyocardial infarction in pigs using AAV-9 and a chimeric vector of AAV-2 and AAV-8 [17-, 37-]. This novel chimeric vector was able to de-target the liver, which is an intriguing feature for more cardiac-specific gene delivery [17-]. Clinical trials with the chimeric vector expressing I-1c are planned to start in 2015 in patients with severe heart failure.

As inhibitor-1 is an upstream regulator of SERCA2a, this molecule also seems to have influence on vascular remodeling. Phenotypic switching of vascular smooth muscle cells from a contractile/quiescent to a proliferative/synthetic phenotype plays a key role in vascular proliferative syndromes such as atherosclerosis [38]. Recently, a synergistic role of I-1 and SERCA2a in the acquisition of the vascular smooth muscle cells contractile phenotype was reported [39-]. Moreover, local I-1c gene transfer in a rat model of carotid injury decreased neointimal formation by preserving vascular smooth muscle cell contractile marker expression, and therefore preventing a vascular smooth muscle cell phenotypic switch [39-]. Hence, in addition to the potential application in HF, I-1c gene transfer appears to be a promising strategy for preventing vascular proliferative disorders.

\section{Inhibition of $G$ protein-coupled receptor kinase-2 by overexpression of $\beta A R K c t$}

$\beta$-adrenergic receptor system dysregulation is another molecular characteristic of HF [40]. In the course of HF, the hyperactivity of the sympathetic nervous system becomes detrimental over time, causing uncoupling and downregulation of $\beta$-adrenergic receptors [41]. G proteincoupled receptor kinase-2 (GRK2), which is markedly upregulated in failing human myocardium plays a key role in the downregulation of $\beta$-adrenergic receptors [40]. Targeted inhibition of GRK2 is possible using a peptide inhibitor known as $\beta$ ARKct. When $\beta$ ARKct is expressed in transgenic mouse models or through adenovirus administration, GRK2 activity is blocked, leading to improved cardiac function [42]. Recently, a large animal preclinical model of HF showed that myocardial AAV-6 delivery of $\beta A R K c t$ reversed ventricular dysfunction, remodeled the heart, and lowered sympathetic outflow of catecholamines, indicating that $\beta$ ARKct administration and GRK2 inhibition are promising therapeutic targets in HF [19].

\section{RNA}

Several studies have attempted to overexpress RNAs using AAV. Successful transduction of RNAs enabled overexpression of therapeutic microRNAs (miRNAs), as well as gene silencing by RNA interference using short-hairpin RNA or small interfering RNA [43,44]. miRNAs are small noncoding RNA that modulate gene expression [45]. Accumulating evidence suggests a critical involvement of miRNAs in the development of cardiovascular disease. Recently, two promising miRNAs have demonstrated therapeutic efficacy in reducing pathological cardiac remodeling using AAV-mediated gene transfer. miR-1 overexpression with AAV-9 resulted in cardiac hypertrophic regression and halted 
functional deterioration in a rat model of transverse aortic constriction [46]. Similarly, miR-378 overexpression was associated with prevention of cardiac hypertrophy and reduced fibrosis in a mouse model of HF [47]. Whether miRNA-based approaches are effective in clinically relevant models currently remains unknown, and future studies are expected to test these promising approaches in larger animals. The use of AAV to increase the level of miRNAs also represents a valuable investigational tool to explore the role of miRNAs in cardiovascular disease pathophysiology. For example, this technology helped to describe the involvement of miR-25 in HF and to identify it as a potentially new therapeutic target [48-].

\section{Other targets}

Catecholaminergic polymorphic ventricular tachycardia (CPVT) is an inherited arrhythmogenic disorder characterized by sudden cardiac death. Drug therapy is still insufficient to provide full protection against cardiac arrest. The disease is related to abnormal calcium storage and release from sarcoplasmic reticulum within the cardiomyocytes, with causative mutations in two key proteins: the ryanodine receptor and the calcium buffering and ryanodine receptor regulatory protein calsequestrin (CASQ2) [49]. In a CPVT knock-in mouse model carrying the CASQ2(R33Q/R33Q)(R33Q) mutation, Denegri et al. [49] recently showed that AAV-9.CASQ2 gene therapy prevents and reverts severe manifestations of CPVT.

Electronic cardiac pacing provides effective treatment for atrial-ventricular block and/or sinus node dysfunction. However, the possibility of replacing wires and power supplies with a biological pacemaker using gene therapy is very attractive [50]. A recent proof-of-concept study demonstrates the feasibility of a gene therapy somatic cell reprogramming strategy for creating a biological pacemaker in a large animal preclinical model of complete heart block [51-]. The gene of the embryonic transcription factor T-box 18 (TBX18) using an adenoviral vector delivery, conferred a sinus-node-like phenotype on adult porcine ventricular myocytes, generating ventricular pacemaker activity that was responsive to autonomic regulation during daily activity [51-]. An earlier study described the feasibility of another gene therapy approach leading to the coexpression of skeletal muscle sodium channel 1 with HCN AQ5 channel 2 in another large animal model [52]. Before a translation to human clinical studies, ongoing efforts focus on safe delivery of long-term biological pacemaker function based on AAV-mediated gene transfer [50].

\section{CLINICAL TRIALS}

A Calcium Upregulation by Percutaneous Administration of Gene Therapy in Cardiac Disease phase 2 trial using AAV-1 to deliver SERCA2a gene therapy by coronary anterograde infusion to patients with chronic HF has been successfully completed. Clinical event rates were significantly lower 3 years after gene transfer in patients receiving highdose AAV-1.SERCA2a compared with those receiving 0.9\% saline [6,7-]. In addition, this study has shown the long-term persistence of the SERCA2a gene in the myocardium up to 31 months after gene transfer [7-]. Further clinical studies are currently underway including a large international study in 250 patients, evaluating whether high-dose AAV-1.SERCA2a (1 $\times 10^{13}$ viral genomes) versus placebo, randomized $1: 1$, is an effective therapy to reduce cardiovascular events in advanced HF [15-]. In Europe, two additional double-blind 
randomized placebo-controlled studies are currently recruiting participants in which the safety and feasibility of AAV-1.SERCA2a therapy is being tested in $24 \mathrm{HF}$ patients that have received a left ventricular assist device (LVAD) for an accepted clinical indication (SERCA-LVADstudy; NCT00534703). Moreover, the impact of AAV-1.SERCA2a therapy on left ventricular remodeling is specifically studied as a primary end point using multimodality cardiac imaging in 44 patients with severe HF (Agent-HF study;

NCT01966887).

\section{CONCLUSION}

Vector modification to enhance and control gene transduction, and improvement in delivery methods, together with identification of novel therapeutic targets will continue to drive the cardiac gene therapy field forward. Increasing numbers of cardiac gene therapy studies using AAV are being conducted in clinically relevant large animal models. This will pave the way to more trials in clinical gene therapy that will ultimately benefit patients with cardiovascular diseases.

\section{Acknowledgements}

The authors would like to thank Lauren Leonardson for valuable editorial assistance.

Financial support and sponsorship

This work is supported by NIH R01 HL117505, HL 119046, a NHLBI Program of Excellence in Nanotechnology (PEN) Award, Contract \# HHSN2682010 00045C, a P50 HL112324, and a Transatlantic Fondation Leducq grant. N.H. was supported by a grant from the French Federation of Cardiology.

\section{REFERENCES AND RECOMMENDED READING}

Papers of particular interest, published within the annual period of review, have been highlighted as:

- of special interest

- of outstanding interest

1. Go AS, Mozaffarian D, Roger VL, et al. Executive summary: heart disease and stroke statistics: 2014 update - a report from the American Heart Association. Circulation. 2014; 129:399-410. [PubMed: 24446411]

2. Yancy CW, Jessup M, Bozkurt B, et al. Writing Committee Members. 2013 ACCF/AHA guideline for the management of heart failure: a report of the American College of Cardiology Foundation/ American Heart Association Task Force on practice guidelines. Circulation. 2013; 128:e240-e327. [PubMed: 23741058]

3. Kawase Y, Ladage D, Hajjar RJ. Rescuing the failing heart by targeted gene transfer. J Am Coll Cardiol. 2011; 57:1169-1180. [PubMed: 21371634]

4. Zinn E, Vandenberghe LH. Adeno-associated virus: fit to serve. Curr Opin Virol. 2014; 8:90-97. [PubMed: 25128609]

5. Jaski BE, Jessup ML, Mancini DM, et al. Calcium upregulation by percutaneous administration of gene therapy in cardiac disease (CUPID Trial), a first-in-human phase 1/2 clinical trial. J Card Fail. 2009; 15:171-181. [PubMed: 19327618]

6. Jessup M, Greenberg B, Mancini D, et al. Calcium Upregulation by Percutaneous Administration of Gene Therapy in Cardiac Disease (CUPID): a phase 2 trial of intracoronary gene therapy of 
sarcoplasmic reticulum Ca2+-ATPase in patients with advanced heart failure. Circulation. 2011; 124:304-313. [PubMed: 21709064]

7. Zsebo K, Yaroshinsky A, Rudy JJ, et al. Long-term effects of AAV1/SERCA2a gene transfer in patients with severe heart failure: analysis of recurrent cardiovascular events and mortality. Circ Res. 2014; 114:101-108. [PubMed: 24065463] This is the first phase 2 clinical trial of AAVmediated gene therapy in heart failure (3 years follow-up results).

8. Atchison RW, Casto BC, Hammon WM. Adenovirus-associated defective virus particles. Science. 1965; 149:754-756. [PubMed: 14325163]

9. Zincarelli C, Soltys S, Rengo G, Rabinowitz JE. Analysis of AAV serotypes 1-9 mediated gene expression and tropism in mice after systemic injection. Mol Ther. 2008; 16:1073-1080. [PubMed: 18414476]

10. Zaiss A-K, Liu Q, Bowen GP, et al. Differential activation of innate immune responses by adenovirus and adeno-associated virus vectors. J Virol. 2002; 76:4580-4590. [PubMed: 11932423]

11. Clark KR, Sferra TJ, Johnson PR. Recombinant adeno-associated viral vectors mediate long-term transgene expression in muscle. Hum Gene Ther. 1997; 8:659-669. [PubMed: 9113506]

12. Zhang Y, Yue Y, Li L, et al. Dual AAV therapy ameliorates exercise-induced muscle injury and functional ischemia in murine models of Duchenne muscular dystrophy. Hum Mol Genet. 2013; 22:3720-3729. [PubMed: 23681067]

13. Koo T, Popplewell L, Athanasopoulos T, Dickson G. Triple trans-splicing adeno-associated virus vectors capable of transferring the coding sequence for full-length dystrophin protein into dystrophic mice. Hum Gene Ther. 2014; 25:98-108. [PubMed: 24191945]

14. Jeune VL, Joergensen JA, Hajjar RJ, Weber T. Preexisting anti-adeno-associated virus antibodies as a challenge in AAV gene therapy. Hum Gene Ther Methods. 2013; 24:59-67. [PubMed: 23442094]

15. Greenberg B, Yaroshinsky A, Zsebo KM, et al. Design of a phase $2 b$ trial of intracoronary administration of AAV1/SERCA2a in patients with advanced heart failure: the CUPID 2 trial (calcium up-regulation by percutaneous administration of gene therapy in cardiac disease phase 2b). JACC Heart Fail. 2014; 2:84-92. [PubMed: 24622121] This is an ongoing large international phase $2 \mathrm{~b}$ study in AAV SERCA2a therapy in HF.

16. Qiao C, Li C, Zhao C, et al. K137R mutation on adeno-associated viral capsids had minimal effect on enhancing gene delivery in vivo. Hum Gene Ther Methods. 2014; 25:33-39. [PubMed: 24116972]

17. Ishikawa K, Fish KM, Tilemann L, et al. Cardiac I-1c overexpression with reengineered aav improves cardiac function in swine ischemic heart failure. Mol Ther. 2014; 22:2038-2045. [PubMed: 25023328] A chimeric AAV vector developed by capsid reengineering effectively transduce the heart for the first time in a clinically relevant large animal model.

18. Chicoine LG, Montgomery CL, Bremer WG, et al. Plasmapheresis eliminates the negative impact of AAV antibodies on microdystrophin gene expression following vascular delivery. Mol Ther. 2014; 22:338-347. [PubMed: 24196577]

19. Raake PWJ, Schlegel P, Ksienzyk J, et al. AAV6.ßARKct cardiac gene therapy ameliorates cardiac function and normalizes the catecholaminergic axis in a clinically relevant large animal heart failure model. Eur Heart J. 2013; 34:1437-1447. [PubMed: 22261894]

20. Katz MG, Fargnoli AS, Williams RD, et al. Safety and efficacy of high-dose adeno-associated virus 9 encoding sarcoplasmic reticulum $\mathrm{Ca}(2+)$ adenosine triphosphatase delivered by molecular cardiac surgery with recirculating delivery in ovine ischemic cardiomyopathy. $\mathrm{J}$ Thorac Cardiovasc Surg. 2014; 148:1065-1072. [PubMed: 25037619] This article describes in a large animal model a promising delivery method for AAV gene therapy.

21. Fargnoli AS, Katz MG, Williams RD, et al. A needleless liquid jet injection delivery method for cardiac gene therapy: a comparative evaluation versus standard routes of delivery reveals enhanced therapeutic retention and cardiac specific gene expression. J Cardiovasc Transl Res. 2014; 7:756-767. [PubMed: 25315468] 
22. Winslow RL, Rice J, Jafri S, et al. Mechanisms of altered excitation-contraction coupling in canine tachycardia-induced heart failure, II: model studies. Circ Res. 1999; 84:571-586. [PubMed: 10082479]

23. Lompré A-M, Hajjar RJ, Harding SE, et al. Ca2+ cycling and new therapeutic approaches for heart failure. Circulation. 2010; 121:822-830. [PubMed: 20124124]

24. Hadri L, Bobe R, Kawase Y, et al. SERCA2a gene transfer enhances eNOS expression and activity in endothelial cells. Mol Ther. 2010; 18:1284-1292. [PubMed: 20461063]

25. Hadri L, Kratlian RG, Benard L, et al. Therapeutic efficacy of AAV1.SERCA2a in monocrotalineinduced pulmonary arterial hypertension. Circulation. 2013; 128:512-523. [PubMed: 23804254] This study reported in mice that pulmonary hypertension is a new promising application of AAV-1 SERCA2a gene therapy.

26. Kho C, Lee A, Jeong D, et al. SUMO1-dependent modulation of SERCA2a in heart failure. Nature. 2011; 477:601-605. [PubMed: 21900893]

27. Schwartz RJ, Yeh ETH. Weighing in on heart failure: the role of SERCA2a SUMOylation. Circ Res. 2012; 110:198-199. [PubMed: 22267837]

28. Lee A, Jeong D, Mitsuyama S, et al. The role of SUMO-1 in cardiac oxidative stress and hypertrophy. Antioxid Redox Signal. 2014; 21:1986-2001. [PubMed: 24893265]

29. Tilemann L, Lee A, Ishikawa K, et al. SUMO-1 gene transfer improves cardiac function in a largeanimal model of heart failure. Sci Transl Med. 2013; 5:211ra159. This is the first large animal study showing the potential efficacy of AAV SUMO-1 therapy in HF.

30. Kraus C, Rohde D, Weidenhammer C, et al. S100A1 in cardiovascular health and disease: closing the gap between basic science and clinical therapy. J Mol Cell Cardiol. 2009; 47:445-455. [PubMed: 19538970]

31. Most P, Seifert H, Gao E, et al. Cardiac S100A1 protein levels determine contractile performance and propensity toward heart failure after myocardial infarction. Circulation. 2006; 114:1258-1268. [PubMed: 16952982]

32. Pleger ST, Shan C, Ksienzyk J, et al. Cardiac AAV9-S100A1 gene therapy rescues postischemic heart failure in a preclinical large animal model. Sci Transl Med. 2011; 3:92ra64.

33. Weber C, Neacsu I, Krautz B, et al. Therapeutic safety of high myocardial expression levels of the molecular inotrope S100A1 in a preclinical heart failure model. Gene Ther. 2014; 21:131-138. [PubMed: 24305416] This study reported S100A1 as a promising target for AAV gene therapy in HF.

34. Haghighi K, Bidwell P, Kranias EG. Phospholamban interactome in cardiac contractility and survival: a new vision of an old friend. J Mol Cell Cardiol. 2014; 77:160-167. [PubMed: 25451386]

35. Kranias EG, Hajjar RJ. Modulation of cardiac contractility by the phospholamban/SERCA2a regulatome. Circ Res. 2012; 110:1646-1660. [PubMed: 22679139]

36. Pathak A, del Monte F, Zhao W, et al. Enhancement of cardiac function and suppression of heart failure progression by inhibition of protein phosphatase 1. Circ Res. 2005; 96:756-766. [PubMed: 15746443]

37. Fish KM, Ladage D, Kawase Y, et al. AAV9.I-1c delivered via direct coronary infusion in a porcine model of heart failure improves contractility and mitigates adverse remodeling. Circ Heart Fail. 2013; 6:310-317. [PubMed: 23271792] This study describes I-1c as a promising target for AAV gene therapy in HF.

38. Dzau VJ, Braun-Dullaeus RC, Sedding DG. Vascular proliferation and atherosclerosis: new perspectives and therapeutic strategies. Nat Med. 2002; 8:1249-1256. [PubMed: 12411952]

39. Lipskaia L, Bobe R, Chen J, et al. Synergistic role of protein phosphatase inhibitor 1 and sarco/ endoplasmic reticulum $\mathrm{Ca} 2+-\mathrm{ATPase}$ in the acquisition of the contractile phenotype of arterial smooth muscle cells. Circulation. 2014; 129:773-785. [PubMed: 24249716] This study underscores the synergistic role of protein phosphatase inhibitor 1 and SERCA2a in vascular diseases.

40. Lohse MJ, Engelhardt S, Eschenhagen T. What is the role of beta-adrenergic signaling in heart failure? Circ Res. 2003; 93:896-906. [PubMed: 14615493] 
41. Brodde OE. Beta-adrenoceptors in cardiac disease. Pharmacol Ther. 1993; 60:405-430. [PubMed: 7915424]

42. Williams ML, Hata JA, Schroder J, et al. Targeted beta-adrenergic receptor kinase (betaARK1) inhibition by gene transfer in failing human hearts. Circulation. 2004; 109:1590-1593. [PubMed: 15051637]

43. Piras BA, O'Connor DM, French BA. Systemic delivery of shRNA by AAV9 provides highly efficient knockdown of ubiquitously expressed GFP in mouse heart, but not liver. PLoS One. 2013; 8:e75894. [PubMed: 24086659]

44. Huang BS, Chen A, Ahmad M, et al. Mineralocorticoid and AT1 receptors in the paraventricular nucleus contribute to sympathetic hyperactivity and cardiac dysfunction in rats post myocardial infarct. J Physiol. 2014; 592(Pt 15):3273-3286. [PubMed: 24951624]

45. Van Rooij E. The art of microRNA research. Circ Res. 2011; 108:219-234. [PubMed: 21252150]

46. Karakikes I, Chaanine AH, Kang S, et al. Therapeutic cardiac-targeted delivery of miR-1 reverses pressure overload-induced cardiac hypertrophy and attenuates pathological remodeling. J Am Heart Assoc. 2013; 2:e000078. [PubMed: 23612897]

47. Ganesan J, Ramanujam D, Sassi Y, et al. MiR-378 controls cardiac hypertrophy by combined repression of mitogen-activated protein kinase pathway factors. Circulation. 2013; 127:20972106. [PubMed: 23625957]

48. Wahlquist C, Jeong D, Rojas-Muñoz A, et al. Inhibition of miR-25 improves cardiac contractility in the failing heart. Nature. 2014; 508:531-535. [PubMed: 24670661] This study describes how AAV technology helped to identify miR25 as a new target for HF therapy.

49. Denegri M, Bongianino R, Lodola F, et al. Single delivery of an adeno-associated viral construct to transfer the CASQ2 gene to knock-in mice affected by catecholaminergic polymorphic ventricular tachycardia is able to cure the disease from birth to advanced age. Circulation. 2014; 129:26732681. [PubMed: 24888331]

50. Boink GJJ, Robinson RB. Gene therapy for restoring heart rhythm. J Cardiovasc Pharmacol Ther. 2014; 19:426-438. [PubMed: 24742766]

51. Hu Y-F, Dawkins JF, Cho HC, et al. Biological pacemaker created by minimally invasive somatic reprogramming in pigs with complete heart block. Sci Transl Med. 2014; 6:245ra94. This is a proof-of-concept study demonstrating the feasibility of a gene therapy somatic cell reprogramming strategy for creating a biological pacemaker.

52. Boink GJJ, Duan L, Nearing BD, et al. HCN2/SkM1 gene transfer into canine left bundle branch induces stable, autonomically responsive biological pacing at physiological heart rates. J Am Coll Cardiol. 2013; 61:1192-1201. [PubMed: 23395072] 


\section{KEY POINTS}

- AAV vectors are safe and confer long-term expression.

- A large clinical trial testing the efficacy of AAV-1. SERCA2a gene therapy is underway.

- Several molecular HF targets such as SUMO-1, S100A1, and $\beta A R K c t$ have been successfully targeted in relevant large animal models and are likely to be clinically evaluated in the near future.

- In addition to HF, preclinical studies suggest the potential utility of AAV gene therapy for arrhythmias and biological heart pacing. 


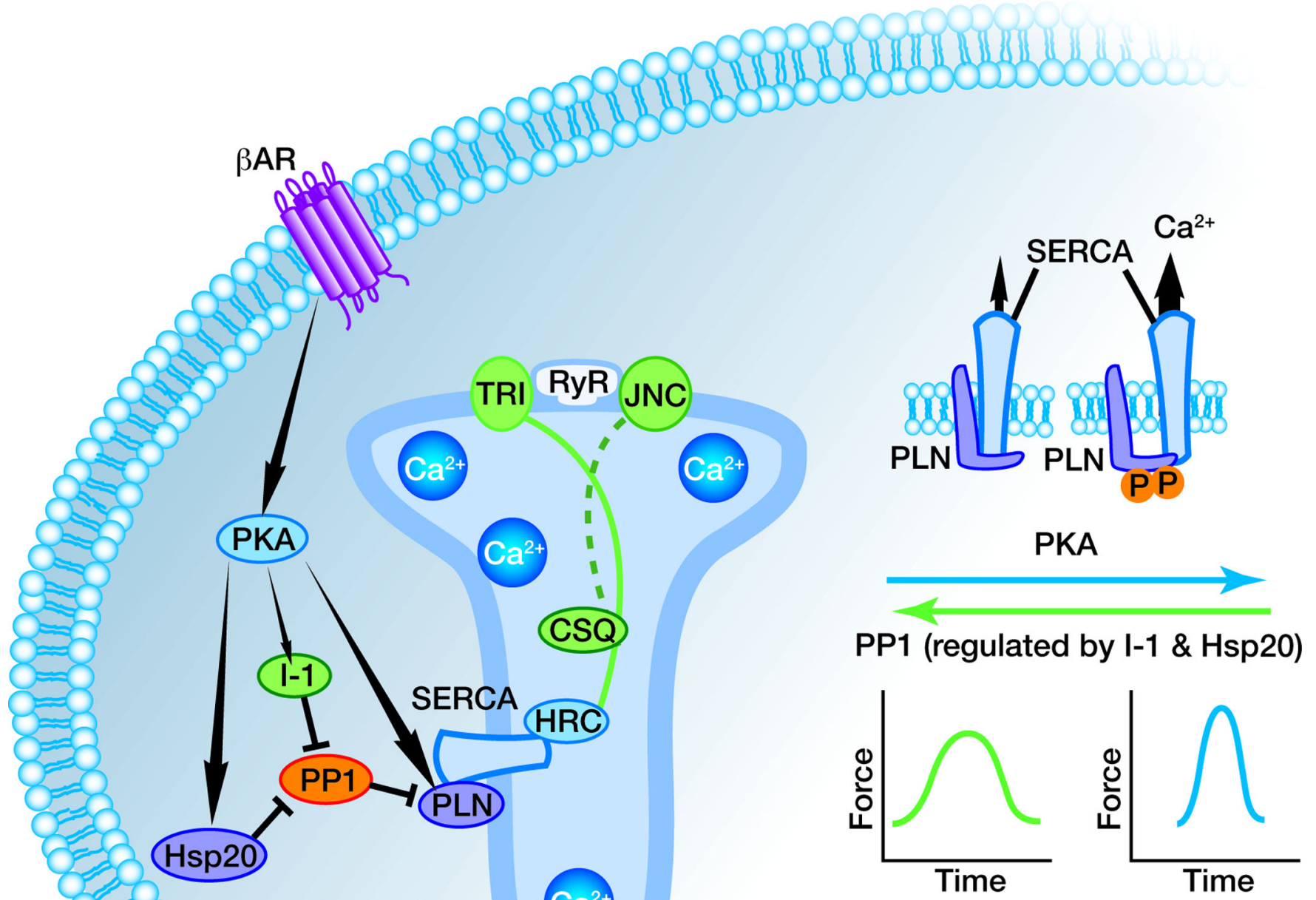

Sarcoplasm

$\mathrm{Ca}^{2+}$ reticulum

FIGURE 1.

Schematic representation of the sarcoplasmic reticulum calcium ATPase (SERCA2a) regulation by protein AQ6 phosphatase 1 inhibitor-1, SERCA2a activity is downregulated by phospholamban. The phosphorylation of phospholamban (PLN) reduces its inhibitor effect on sarcoplasmic reticulum calcium ATPase (SERCA2a) pump. During $\beta$-adrenergic stimulation PLN is phosphorylated by protein kinase A (PKA). PLN dephosphorylation is due to protein phosphatase 1 (PP1). Inhibition of PP1 by inhibitor-1 (I-1) leads to enhance the phosphorylation of PLN that results in increased SERCA2a activity. PP1 is also regulated by heat shock protein 20 (Hsp) 20. The sarcoplasmic reticulum calcium $\left(\mathrm{Ca}^{2+}\right)$ 
release complex included ryanodine receptor (RyR), triadin (TRI), junctin (JNC) and calsequestrin (CSQ). Reproduced with permission from [35]. 